\title{
An Integrated Approach for Management of Thrips tabaci Lindeman in Rabi Onion under Gangetic Plains of West Bengal, India
}

\author{
Maimom Soniya Devi ${ }^{1 *}$ and Kusal Roy ${ }^{2}$ \\ ${ }^{1}$ Department of Entomology, Lovely Professional University, \\ Phagwara-144411, Punjab, India \\ ${ }^{2}$ Department of Agricultural Entomology, Bidhan Chandra Krishi Viswavidyalaya Mohanpur- \\ 741252, Nadia, West Bengal, India \\ *Corresponding author
}

\begin{tabular}{|c|c|}
\hline & A B S T R A C T \\
\hline Keywords & \multirow{4}{*}{$\begin{array}{l}\text { An experiment was conducted for two consecutive years during rabi season of 2014-15 } \\
\text { and } 2015-16 \text { to manage the onion thrips using three treatment modules viz., M1- IPM } \\
\text { module, M2- Farmers' practice and M3-Untreated control. It has been observed that the } \\
\text { IPM module was the best in achieving the maximum leaf growth and bulb yield in both the } \\
\text { years ( } 2014-15 \text { and } 2015-16 \text { ) followed by farmers' practice module. Adoption of IPM } \\
\text { module (comprised of wheat and maize as barrier crops, seed treatment with imidacloprid } \\
70 \text { WS and spraying of Beauveria bassiana } 10 \text { SC and spinosad } 45 \text { SC at ETL) kept the } \\
\text { plant less infested by the T. tabaci. An avoidable yield loss of onion bulb due to infestation } \\
\text { of } T \text {. tabaci was estimated as } 18.98 \% \text {. The incremental benefit cost ratio (IBCR) was } \\
\text { found more in IPM module than the farmers' practice module. The maximum return per } \\
\text { rupee investment for managing thrips in rabi onion was observed with IPM module being, } \\
\text { Rs. } 6.65 \text {. }\end{array}$} \\
\hline $\begin{array}{l}\text { Avoidable yield loss, } \\
\text { IPM module, } \\
\text { Incremental benefit } \\
\text { cost ratio, Onion } \\
\text { thrips, Blue sticky } \\
\text { trap, Population }\end{array}$ & \\
\hline Article Info & \\
\hline $\begin{array}{l}\text { Accepted: } \\
\text { 20 June } 2018 \\
\text { Available Online: } \\
\text { 10 July } 2018\end{array}$ & \\
\hline
\end{tabular}

\section{Introduction}

Onion (Allium cepa L.) is one of the important commercial vegetable crops grown in India. It is preferred by people for its distinctive flavour and is relished mostly as "green" for salad and Indian cuisine is incomplete without onion. There are several factors that lead to the low productivity of onion. Among these, one of the major constraints is insect pest. Among the insect pests, onion thrips, Thrips tabaci Lindeman has become a global pest of increasing concern in the past three decades
(Diaz-Monatano et al., 2011). Thrips is a regular and potential pest of onion and cause considerable yield losses as high as $90 \%$ in terms of quality and quantity (Gupta et al., 1984; Darmasena, 1998; Sudharma and Nair, 1999). To tackle this sucking pest menace, farmers are extensively using different types of insecticides. However, a repeated application of chemicals is not a desirable practice, as this could lead to undesirable resistance problems. To avoid further resistance in this pest, different non chemical methods need to be evaluated. Multi-pronged 
pest management strategies that boost onion plant health and tolerance to thrips, in addition to suppressing thrips densities, have to be developed for the most sustainable and economically viable thrips management tactics.

\section{Materials and Methods}

The experiment was conducted for two consecutive years during rabi season of 201415 and 2015-16 at the C-Block Farm of Bidhan Chandra Krishi Viswavidyalaya, Kalyani, Nadia, West Bengal. Onion cultivar Sukhsagar was used for the experiment. Experiment was planned in Randomized Complete Block Design. Each plot was measuring $2.1 \mathrm{~m} \times 1.95 \mathrm{~m}$ (4.095 sq. $\mathrm{m}$ ). The crop was raised following recommended package of practices. Thirty days old seedlings were transplanted in the main field at $15 \mathrm{~cm} \times$ $15 \mathrm{~cm}$ spacing. There were three treatment modules viz., $\mathrm{M}_{1}$-IPM module, $\mathrm{M}_{2}$ - Farmers' practice and $\mathrm{M}_{3}$-Untreated control which were replicated seven times.

\section{Treatment details}

The details of the modules are as follows:

\section{$M_{1}$ : IPM module}

Planting of barrier crops -two continuous inner rows of wheat (cv. Purbali) followed by two continuous outer rows of maize (cv. Kishan)were sown@113 kg/ha and $23 \mathrm{~kg} / \mathrm{ha}$, respectively on all 4 sides outside the plot covering 1.78 sq. m area $(20 \mathrm{~cm}$ barrier crops area on all four sides of a $2.1 \mathrm{~m} \times 1.95 \mathrm{~m}$ plot) at 20 days prior to planting of onion. Hence, in IPM module, out of 1 hectare (ten thousand sq. m) cultivable area approximately 6970 sq. $\mathrm{m}$ area will be available for planting of onion crop and remaining 3030 sq. m area (approx.) will be consumed for planting of barrier crops. Onion seeds were treated with imidacloprid 70WS@2.45g a.i./kg of seed before sowing in the nursery (http://skuast.org).

Spraying was initiated when thrips population reach economic threshold level (ETL), $1^{\text {st }}$ spray was given with Beauveria bassiana 10 SC@ $75 \mathrm{~g}$ a.i./ha and $2^{\text {nd }}$ spray was given with spinosad 45SC @ 73g a.i./ha.

ETL was considered as 30 thrips/plant (http://www.dogr.res.in).

Time of spray- 72 DAP and 86 DAP

\section{$M_{2}$ : Farmers' practice}

- Insecticidal spray was given at 21 days interval starting from the appearance of onion thrips. Acetamiprid 20SP @ 35g a.i./ha as $1^{\text {st }}$ spray, imidacloprid 17.8SL@ $35 \mathrm{~g}$ a.i./ha as $2^{\text {nd }}$ spray and acephate 75SP @ 280g a.i./ha as $3^{\text {rd }}$ spray were used.

Time of spray- 30 DAP, 51 DAP and 72 DAP

\section{$M_{3}$ : Untreated control}

(did not receive any insecticidal spray)

\section{Observations recorded}

Population of thrips was monitored by using blue sticky trap at weekly interval. As soon as the thrips appear in the field, population of the thrips was counted at weekly interval from 10 randomly selected tagged plants per plot. Thrips which were stuck on the blue sticky traps were also counted from the ten square grids using hand held magnifying lens. Fresh and dry weight of bulb and number of bulbs per plot were also recorded.

Onion equivalent yield (OEY) of wheat and maize grown as border crop in the IPM module plots was also calculated considering the local market price following the formula as stated by Talukder et al., (2015) and Anjaneyulu et al., (1982). 


$\operatorname{OEY}\left(\mathbf{q} /\right.$ ha) $=$ Onion bulb yield $(\mathbf{q} / \mathbf{h a})+\frac{\begin{array}{c}\text { Grain yield of wheat }(\mathbf{q} / \mathbf{h a}) \times \text { Price of } \\ \text { wheat grain }(\mathbf{R s} . / \mathbf{q})\end{array}}{\text { Price of onion }(\text { Rs. } / \mathbf{q})}+\frac{\begin{array}{c}\text { Grain yield of maize }(\mathbf{q} / \mathbf{h a}) \times \text { Price of maize } \\ \text { grain }(\text { Rs./q) }\end{array}}{\text { Price of onion }(\text { Rs. } / \mathbf{q})}$

\section{Statistical analysis}

Analysis of variance was done according to RCBD at 5\% level of probability (Gomez and Gomez, 1984). Assessment of avoidable loss of bulb yield due to infestation of T. tabaci in onion cv. Sukhsagar was worked out using the concept given by Roy et al., 2008 and Basavaraju et al., 2009.

\% avoidable yield loss

(Dry bulb yield in IPM module - Dry bulb yield in untreated control)

Dry bulb yield in IPM module $\times 100$

\section{Results and Discussion}

Effect of management modules on population of thrips on onion crop during rabi, 2014-15 and 2015-16

The effect of management modules on population of thrips of onion crop during rabi, 2014-15 and 2015-16 are presented here (Table 1-4). Data indicated that there were no populations of thrips in IPM module $\left(\mathrm{M}_{1}\right)$ till 37 DAP in both the years of experimentation. Thrips populations were detected first on the onion plant at 30 DAP (Table 1) and the spraying of acetamiprid 20SP @ 35g a.i./ha was initiated on that day at the plots receiving farmers' practice module of thrips management.

At 30 DAP, in 2015-16 none of the modules showed any significant differences among them with regard to number of thrips per plant while, significant differences were observed in 2014-15.

The non-significant effect on population of thrips was noted at the time of their initiation i.e. during 30 DAP. Similar observation was earlier cited by Tripathy et al., (2013), though pooled data (2014-15 and 2015-16) of thrips population at 30 DAP revealed significant variations among treatment modules.
Significant differences in thrips population per plant had been observed among modules from 37 DAP to 100 DAP (Table 1-4) in both the years of the study except second year where thrips population did not reveal significant differences at 58 DAP and 86 DAP (Table 1). Pooled data however exhibited significant differences on occurrences of thrips per plant (Table 1-4).

Thrips populations were always recorded maximum with untreated control (Table 1-4). The number of thrips per plant was minimum with IPM module until 58 DAP from their emergence on the onion crop (Table 2). The better efficacy upto 58DAP in IPM module might be due to border crop effect of both wheat and maize. As thrips are weak fliers and carried by wind, planting barrier crop like maize and wheat could effectively block or reduce adult thrips reaching onion plant (Tripathy et al., 2013).

The use of barrier crops ( 2 rows of maize, the outer rows +2 rows of wheat, the inner rows) as advocated in the present IPM module was also earlier adopted by Srinivas and Lawande (2006). They observed very effective blocking of adult thrips by the barrier crops which are in conformity with the present findings and showing the usefulness of barrier crop in restricting the entry of onion thrips from 
adjoining areas. Thereafter, from 65 DAP to 72 DAP, thrips populations per plant in IPM module plots took upper hand over plots receiving farmers' practice module of thrips management tactics $\left(\mathrm{M}_{2}\right)$ (Table 2-3). Module comprising IPM tactics could able to restrict the buildup of $T$. tabaci population below economic threshold level till 65 DAP. At 72 DAP, when $T$. tabaci population crossed the ETL (30thrips/plants), the $1^{\text {st }}$ foliar spray of Beauveria bassiana 10SC @ 75g a.i./ha was given in IPM module plots. Subsequent two sprays of imidacloprid 17.8SL @ 35g a.i./ha as $2^{\text {nd }}$ spray and acephate 75SP @ 280g a.i./ha as $3^{\text {rd }}$ spray were advocated in farmers' practice module at 51 DAP and 72 DAP, respectively.

After a first peak of $T$. tabaci population at 72 DAP, a second peak of $T$. tabaci population at 86 DAP were observed (Table 3). Hence, a second round of foliar spray with spinosad 45SC @ 73g a.i./ha was given in IPM module plots. No additional sprays were given in farmers' practice plots. Insecticidal sprays advocated in farmers' practice module could not able to check the buildup of $T$. tabaci population much over the period.

After initiation of insecticidal spray in IPM module at 72 DAP, thrips population became significantly low at 79 DAP as compared to other modules in both the years (Table 3 ).

Annamalai et al., (2016) evaluated bioefficacy of commercial formulation of $B$. bassiana (Myco-Jaal) @ $1 \times 10^{8}$ spores $/ \mathrm{ml}$ which showed $80.90 \%$ mortality of $T$. tabaci. B. bassiana was most effective when used early at economic threshold level, before large thrips populations have built up. Beauveria species is usually used as a contact mycoinsecticide but survives a relatively short period of time when exposed on a leaf surface (Brian, 2006). In the present study, a significant reduction in thrips population below ETL was observed in IPM module just after initial spray of $B$. bassiana and sometimes later, an increment in population over ETL was noted. This finding can suitably justify the previous opinion of Annamalai et al., 2016 and Brian, 2006.

Second round of spray at 86 DAP with spinosad 45SC @ 73g a.i./ha in IPM module plots sharply declined the T. tabaci population at 93 DAP (Table 4). Spinosad @ 0.0135\% was the most toxic against adult thrips of onion (Mahmoud et al., 2007; Panse et al., 2012). Pooled data also disclosed similar trend. Later, all plots had general reduction of thrips population as the crop progressed towards senescence (Table 4). The findings are also in conformity with the study conducted by Tripathy et al., (2013) who reported that significant reduction of thrips population both in IPM module and farmers' practices over the untreated plot.

The adult population of $T$. tabaci was also monitored by using blue sticky trap @ 1/4.095 sq. $m$ area of plot in all the modules. The population of thrips trapped in blue sticky trap was confirmed as $T$. tabaci by microscopic observation (Plate 5). In both the years of study (2014-15), no population of $T$. tabaci was observed in $\mathrm{M}_{1}$ module at 23 DAP (Table 5). Occurrence of T. tabaci on the blue sticky trap was first detected at 23 DAP in untreated control and farmers' practice modules in the $1^{\text {st }}$ and $2^{\text {nd }}$ year, respectively (Table 5). The three modules didn't show any significant differences in number of thrips caught per square inch area of blue sticky trap as observed from pooled data at 23 DAP (Table $5)$.

The number of thrips caught in blue sticky trap at 30 DAP did not reveal any significant variations in both the years. The pooled data exhibited significantly least number of thrips population in IPM module plots at 30 DAP 
being, $0.1 /$ sq. inch area of trap whereas, in farmers' practice and in untreated control plots mean thrips population was $0.2 /$ sq. inch area of trap at 30 DAP (Table 5).

Starting from 37 DAP to 100 DAP, significant differences in trapping of thrips on blue sticky trap among management modules were observed during the study period of 2014-15 and 2015-16 (Table 5-8). Pooled data of two years' observation revealed significant low catch of T. tabaci in IPM module $\left(\mathrm{M}_{1}\right)$ ranging from 0.1-7.2/ sq. inch area of blue sticky trap from 37 to 58 DAP (Table 5, 6).

The numbers of onion thrips caught during 37 to 58 DAP were usually more in the farmers' practice and untreated control modules. Maximum population of thrips trapped during 37 to 58 DAP was ranged from 0.5-10.7/sq. inch area of blue sticky trap in untreated control module (Table 5, 6).

Catches of adult stages of T. tabaci population in blue sticky trap were more in IPM module as compared to farmers' practice module at 65 DAP and 72 DAP (Table 7). Trapping of thrips on blue sticky trap depends on the availability of their adult stages in the crop field. It had been viewed from the pooled data that irrespective of modules catches of $T$. tabaci population were more during 65 DAP to 86 DAP except 79 DAP (Table 7, 8). At 79 DAP, number of thrips caught in trap were low in IPM and farmers' practice modules whereas, in untreated control number of thrips catches in trap increased gradually from 65 86 DAP without any interruption in between (based on pooled data). This reduction in adult thrips population may be due to application of Beauveria bassiana 10SC@75g a.i./ha in IPM module plots and acetamiprid 20SP @ $35 \mathrm{~g}$ a.i./ha in farmers' practice module. It has also been observed from a laboratory study during rabi season that egg to adult stage of $T$. tabaci on onion could be completed within 1420 days. Hence, the variable occurrences of adult population of thrips at different time (as confirmed from trap catches) are a sign of the presence of several overlapping generations of thrips in the onion crop field.

In spite of three round of insecticidal spray, the plots in farmers' practice module always had more population of $T$. tabaci in comparison to IPM module except at 65 DAP (Table 7). Crops which are without any barrier may be reinfested by $T$. tabaci population from the adjoining onion crop fields. Possibility of such type of instances cannot be overruled here. Higher trapping of thrips in the farmers' practice module and in untreated control module as evidenced by the data signifies the aforementioned statement.

A marked decline in population of adult thrips in IPM module were observed at 93 days after planting as the plots received a second round of foliar spray with spinosad 45SC @ 73g a.i./ha at 86 DAP (Table 8). Afterwards, a general decline of adult thrips population was observed in all plots as the crop progressed towards senescence (Table 8).

Effect of thrips management modules on yield of onion during rabi, 2014-15 and 2015-16

The production of number of onion bulbs during rabi season of 2014-15 and 2015-16 were significantly more in IPM module as compared to untreated control being, 122/plot and 184/plot, respectively for the $1^{\text {st }}$ and $2^{\text {nd }}$ year (Table 9). Farmers' practice module was followed thereafter in production of bulbs being, 119 bulbs/plot and 179 bulbs/plot, respectively for the $1^{\text {st }}$ and $2^{\text {nd }}$ year (Table 9). Both the mentioned modules were at par with each other.

The pooled data of two years experimentation showed more number of bulbs in $\mathrm{M}_{1}$ followed by $\mathrm{M}_{2}$ being, 153/plot and 149/plot, respectively (Table 9). 
Table.1 Effect of management modules on thrips population of onion during rabi, 2014-15 and 2015-16 (Mean of 7 replications)

\begin{tabular}{|c|c|c|c|c|c|c|c|c|c|}
\hline \multirow[t]{3}{*}{ Modules } & \multicolumn{9}{|c|}{ Population of thrips per plant } \\
\hline & \multicolumn{3}{|c|}{ 30DAP } & \multicolumn{3}{|c|}{ 37DAP } & \multicolumn{3}{|c|}{ 44DAP } \\
\hline & $\begin{array}{c}\text { 2014- } \\
15\end{array}$ & $\begin{array}{c}2015- \\
16\end{array}$ & Pooled & $\begin{array}{c}2014- \\
15\end{array}$ & $\begin{array}{c}2015- \\
16\end{array}$ & Pooled & $\begin{array}{c}\text { 2014- } \\
15\end{array}$ & $\begin{array}{c}\text { 2015- } \\
16\end{array}$ & Pooled \\
\hline $\mathbf{M}_{1}$ & $\begin{array}{c}0.7 \\
(0.0)\end{array}$ & $\begin{array}{c}0.7 \\
(0.0)\end{array}$ & $\begin{array}{c}0.7 \\
(0.0)\end{array}$ & $\begin{array}{c}0.7 \\
(0.0)\end{array}$ & $\begin{array}{c}0.7 \\
(0.0)\end{array}$ & $\begin{array}{c}0.7 \\
(0.0)\end{array}$ & $\begin{array}{c}0.7 \\
(0.0)\end{array}$ & $\begin{array}{c}0.9 \\
(0.4)\end{array}$ & $\begin{array}{c}0.8 \\
(0.2)\end{array}$ \\
\hline $\mathbf{M}_{2}$ & $\begin{array}{c}0.8 \\
(0.1)\end{array}$ & $\begin{array}{c}0.7 \\
(0.1)\end{array}$ & $\begin{array}{c}0.8 \\
(0.1)\end{array}$ & $\begin{array}{c}1.0 \\
(0.7)\end{array}$ & $\begin{array}{c}0.9 \\
(0.3)\end{array}$ & $\begin{array}{c}1.0 \\
(0.5)\end{array}$ & $\begin{array}{c}1.3 \\
(1.1)\end{array}$ & $\begin{array}{c}1.2 \\
(0.9)\end{array}$ & $\begin{array}{c}1.2 \\
(1.0)\end{array}$ \\
\hline $\mathbf{M}_{3}$ & $\begin{array}{c}0.8 \\
(0.2)\end{array}$ & $\begin{array}{c}0.8 \\
(0.1)\end{array}$ & $\begin{array}{c}08 \\
(0.1)\end{array}$ & $\begin{array}{c}1.1 \\
(0.7)\end{array}$ & $\begin{array}{c}0.9 \\
(0.4)\end{array}$ & $\begin{array}{c}1.0 \\
(0.5)\end{array}$ & $\begin{array}{c}1.3 \\
(1.2)\end{array}$ & $\begin{array}{c}1.1 \\
(0.8)\end{array}$ & $\begin{array}{c}1.2 \\
(1.0)\end{array}$ \\
\hline $\operatorname{SEm}( \pm)$ & 0.01 & 0.02 & 0.01 & 0.06 & 0.03 & 0.03 & 0.05 & 0.04 & 0.03 \\
\hline LSD (0.05) & 0.03 & NS & $\mathbf{0 . 0 3}$ & 0.18 & 0.09 & 0.09 & 0.15 & 0.12 & 0.09 \\
\hline & $\begin{array}{c}\text { SEm } \\
( \pm)\end{array}$ & $\begin{array}{c}\text { LSD } \\
(\mathbf{0 . 0 5})\end{array}$ & & $\begin{array}{c}\text { SEm } \\
( \pm)\end{array}$ & $\begin{array}{c}\text { LSD } \\
(\mathbf{0 . 0 5})\end{array}$ & & $\begin{array}{c}\text { SEm } \\
( \pm)\end{array}$ & $\begin{array}{c}\text { LSD } \\
(\mathbf{0 . 0 5})\end{array}$ & \\
\hline Year & 0.01 & NS & & 0.03 & 0.09 & & 0.03 & NS & \\
\hline Year $\times$ Module & 0.02 & NS & & 0.05 & NS & & 0.05 & 0.15 & \\
\hline
\end{tabular}

Table.2 Effect of management modules on thrips population of onion during rabi, 2014-15 and 2015-16 (Mean of 7 replications)

\begin{tabular}{|c|c|c|c|c|c|c|c|c|c|}
\hline \multirow[t]{3}{*}{ Modules } & \multicolumn{9}{|c|}{ Population of thrips per plant } \\
\hline & \multicolumn{3}{|c|}{ 51DAP } & \multicolumn{3}{|c|}{ 58DAP } & \multicolumn{3}{|c|}{ 65DAP } \\
\hline & $\begin{array}{c}2014- \\
15\end{array}$ & $\begin{array}{c}2015- \\
16\end{array}$ & Pooled & $\begin{array}{c}2014- \\
15\end{array}$ & $\begin{array}{c}2015- \\
16\end{array}$ & Pooled & $\begin{array}{c}2014- \\
15\end{array}$ & $\begin{array}{c}2015- \\
16\end{array}$ & Pooled \\
\hline $\mathbf{M}_{1}$ & $\begin{array}{c}0.8 \\
(0.2)\end{array}$ & $\begin{array}{c}2.0 \\
(3.5)\end{array}$ & $\begin{array}{c}1.4 \\
(1.9)\end{array}$ & $\begin{array}{c}2.8 \\
(7.5)\end{array}$ & $\begin{array}{c}2.8 \\
(7.6)\end{array}$ & $\begin{array}{c}2.8 \\
(7.5)\end{array}$ & $\begin{array}{c}3.8 \\
(13.7)\end{array}$ & $\begin{array}{c}4.3 \\
(18.2)\end{array}$ & $\begin{array}{c}4.0 \\
(16.0)\end{array}$ \\
\hline $\mathbf{M}_{2}$ & $\begin{array}{c}1.9 \\
(3.3)\end{array}$ & $\begin{array}{c}1.3 \\
(1.3)\end{array}$ & $\begin{array}{c}1.6 \\
(2.3)\end{array}$ & $\begin{array}{c}3.6 \\
(12.2)\end{array}$ & $\begin{array}{c}2.8 \\
(7.2)\end{array}$ & $\begin{array}{c}3.2 \\
(9.7)\end{array}$ & $\begin{array}{c}3.3 \\
(10.5)\end{array}$ & $\begin{array}{c}3.0 \\
(8.8)\end{array}$ & $\begin{array}{c}3.2 \\
(9.6)\end{array}$ \\
\hline $\mathbf{M}_{3}$ & $\begin{array}{c}1.8 \\
(2.9)\end{array}$ & $\begin{array}{c}2.1 \\
(3.8)\end{array}$ & $\begin{array}{c}1.9 \\
(3.3)\end{array}$ & $\begin{array}{c}3.7 \\
(13.4)\end{array}$ & $\begin{array}{c}2.8 \\
(7.6)\end{array}$ & $\begin{array}{c}3.3 \\
(10.5)\end{array}$ & $\begin{array}{c}4.5 \\
(19.4)\end{array}$ & $\begin{array}{c}4.5 \\
(19.7)\end{array}$ & $\begin{array}{c}4.5 \\
(19.6)\end{array}$ \\
\hline $\operatorname{SEm}( \pm)$ & 0.05 & 0.05 & 0.04 & 0.07 & 0.06 & 0.05 & 0.05 & 0.09 & 0.05 \\
\hline LSD (0.05) & 0.15 & 0.15 & 0.12 & 0.26 & NS & 0.15 & 0.15 & 0.28 & 0.15 \\
\hline & $\begin{array}{c}\text { SEm } \\
( \pm)\end{array}$ & $\begin{array}{c}\text { LSD } \\
(\mathbf{0 . 0 5})\end{array}$ & & $\begin{array}{c}\text { SEm } \\
( \pm)\end{array}$ & $\begin{array}{c}\text { LSD } \\
(\mathbf{0 . 0 5})\end{array}$ & & $\begin{array}{c}\text { SEm } \\
( \pm)\end{array}$ & $\begin{array}{c}\text { LSD } \\
(\mathbf{0 . 0 5})\end{array}$ & \\
\hline Year & 0.03 & 0.09 & & 0.05 & 0.15 & & 0.03 & 0.09 & \\
\hline Year $\times$ Module & 0.05 & 0.15 & & 0.07 & 0.20 & & 0.07 & 0.21 & \\
\hline
\end{tabular}


Table.3 Effect of management modules on thrips population of onion during rabi, 2014-15 and $2015-16$ (Mean of 7 replications)

\begin{tabular}{|c|c|c|c|c|c|c|c|c|c|}
\hline \multirow[t]{3}{*}{ Modules } & \multicolumn{9}{|c|}{ Population of thrips per plant } \\
\hline & \multicolumn{3}{|c|}{ 72DAP } & \multicolumn{3}{|c|}{ 79DAP } & \multicolumn{3}{|c|}{ 86DAP } \\
\hline & $\begin{array}{c}2014- \\
15\end{array}$ & $\begin{array}{c}2015- \\
16\end{array}$ & Pooled & $\begin{array}{c}2014- \\
15\end{array}$ & $\begin{array}{c}2015- \\
16\end{array}$ & Pooled & $\begin{array}{c}2014- \\
15\end{array}$ & $\begin{array}{c}2015- \\
16\end{array}$ & Pooled \\
\hline $\mathrm{M}_{1}$ & $\begin{array}{c}5.5 \\
(30.3)\end{array}$ & $\begin{array}{c}5.5 \\
(30.2)\end{array}$ & $\begin{array}{c}5.5 \\
(30.3)\end{array}$ & $\begin{array}{c}4.3 \\
(17.9)\end{array}$ & $\begin{array}{c}3.5 \\
(12.1)\end{array}$ & $\begin{array}{c}3.9 \\
(15.0)\end{array}$ & $\begin{array}{c}5.5 \\
(30.4)\end{array}$ & $\begin{array}{c}5.5 \\
(30.0)\end{array}$ & $\begin{array}{c}5.5 \\
(30.2)\end{array}$ \\
\hline $\mathrm{M}_{2}$ & $\begin{array}{c}4.4 \\
(18.5)\end{array}$ & $\begin{array}{c}4.2 \\
(16.8)\end{array}$ & $\begin{array}{c}4.4 \\
(17.7)\end{array}$ & $\begin{array}{c}3.9 \\
(14.7)\end{array}$ & $\begin{array}{c}4.5 \\
(19.9)\end{array}$ & $\begin{array}{c}4.2 \\
(17.3)\end{array}$ & $\begin{array}{c}5.7 \\
(31.9)\end{array}$ & $\begin{array}{c}5.6 \\
(31.0)\end{array}$ & $\begin{array}{c}5.6 \\
(31.5)\end{array}$ \\
\hline $\mathrm{M}_{3}$ & $\begin{array}{c}5.6 \\
(31.3)\end{array}$ & $\begin{array}{c}6.3 \\
(38.8)\end{array}$ & $\begin{array}{c}5.9 \\
(35.0)\end{array}$ & $\begin{array}{c}5.9 \\
(34.9)\end{array}$ & $\begin{array}{c}6.1 \\
(36.5)\end{array}$ & $\begin{array}{c}6.0 \\
(35.7)\end{array}$ & $\begin{array}{c}6.2 \\
(37.9)\end{array}$ & $\begin{array}{c}5.7 \\
(32.3)\end{array}$ & $\begin{array}{c}6.0 \\
(35.1)\end{array}$ \\
\hline $\operatorname{SEm}( \pm)$ & 0.05 & 0.10 & 0.05 & 0.08 & 0.10 & 0.06 & 0.08 & 0.12 & 0.07 \\
\hline \multirow[t]{2}{*}{$\operatorname{LSD}(0.05)$} & 0.15 & 0.31 & 0.15 & 0.25 & 0.31 & 0.18 & 0.25 & NS & 0.22 \\
\hline & $\begin{array}{c}\text { SEm } \\
( \pm)\end{array}$ & $\begin{array}{c}\text { LSD } \\
(0.05)\end{array}$ & & $\begin{array}{c}\text { SEm } \\
( \pm)\end{array}$ & $\begin{array}{c}\text { LSD } \\
(0.05)\end{array}$ & & $\begin{array}{c}\text { SEm } \\
( \pm)\end{array}$ & $\begin{array}{l}\text { LSD } \\
(0.05)\end{array}$ & \\
\hline Year & 0.05 & NS & & 0.04 & NS & & 0.02 & 0.06 & \\
\hline Year $\times$ Module & 0.08 & 0.23 & & 0.09 & 0.26 & & 0.10 & NS & \\
\hline
\end{tabular}

Note: Data in the parentheses indicate original values. Data shown in the table are $(x+0.5)$ square root transformed values. $\mathrm{M}_{1}$ : IPM module, $\mathrm{M}_{2}$ : Farmers' practices and $\mathrm{M}_{3}$ : Control

Table.4 Effect of management modules on thrips population of onion during rabi, 2014-15 and 2015-16 (Mean of 7 replications)

\begin{tabular}{|c|c|c|c|c|c|c|}
\hline \multirow[t]{3}{*}{ Modules } & \multicolumn{6}{|c|}{ Population of thrips per plant } \\
\hline & \multicolumn{3}{|c|}{ 93DAP } & \multicolumn{3}{|c|}{ 100DAP } \\
\hline & 2014-15 & 2015-16 & Pooled & 2014-15 & 2015-16 & Pooled \\
\hline $\mathbf{M}_{1}$ & $\begin{array}{c}3.2 \\
(9.8)\end{array}$ & $\begin{array}{c}3.3 \\
(10.2)\end{array}$ & $\begin{array}{c}3.2 \\
(10.0)\end{array}$ & $\begin{array}{c}3.0 \\
(8.6)\end{array}$ & $\begin{array}{c}2.6 \\
(6.5)\end{array}$ & $\begin{array}{c}2.8 \\
(7.5)\end{array}$ \\
\hline $\mathbf{M}_{2}$ & $\begin{array}{c}4.2 \\
(17.2)\end{array}$ & $\begin{array}{c}4.5 \\
(20.0)\end{array}$ & $\begin{array}{c}4.4 \\
(18.6)\end{array}$ & $\begin{array}{c}3.7 \\
(13.2)\end{array}$ & $\begin{array}{c}3.3 \\
(10.7)\end{array}$ & $\begin{array}{c}3.5 \\
(12.0)\end{array}$ \\
\hline $\mathbf{M}_{3}$ & $\begin{array}{c}6.2 \\
(37.7)\end{array}$ & $\begin{array}{c}5.2 \\
(26.9)\end{array}$ & $\begin{array}{c}5.7 \\
(32.2)\end{array}$ & $\begin{array}{c}5.3 \\
(27.8)\end{array}$ & $\begin{array}{c}4.5 \\
(20.5)\end{array}$ & $\begin{array}{c}4.9 \\
(24.1)\end{array}$ \\
\hline $\operatorname{SEm}( \pm)$ & 0.11 & 0.13 & 0.08 & 0.13 & 0.18 & 0.11 \\
\hline LSD (0.05) & 0.34 & 0.40 & 0.25 & 0.40 & 0.55 & 0.34 \\
\hline & $\operatorname{SEm}( \pm)$ & LSD (0.05) & & $\operatorname{SEm}( \pm)$ & LSD (0.05) & \\
\hline Year & 0.05 & 0.15 & & 0.04 & 0.12 & \\
\hline Year×Module & 0.12 & 0.35 & & 0.16 & NS & \\
\hline
\end{tabular}

Note: Data in the parentheses indicate original values. Data shown in the table are $(x+0.5)$ square root transformed values. $\mathrm{M}_{1}$ : IPM module, $\mathrm{M}_{2}$ : Farmers' practices and $\mathrm{M}_{3}$ : Control 
Table.5 Effect of management modules on population of thrips per square inch of blue sticky trap installed in onion crop field during rabi, 2014-15 and 2015-16

\begin{tabular}{|c|c|c|c|c|c|c|c|c|c|}
\hline \multirow[t]{3}{*}{ Modules } & \multicolumn{9}{|c|}{ Population of $T$. tabaci per square inch area of blue sticky trap } \\
\hline & \multicolumn{3}{|c|}{ 23DAP } & \multicolumn{3}{|c|}{ 30DAP } & \multicolumn{3}{|c|}{ 37DAP } \\
\hline & $\begin{array}{c}2014- \\
15\end{array}$ & $\begin{array}{c}2015- \\
16\end{array}$ & Pooled & $\begin{array}{c}2014- \\
15\end{array}$ & $\begin{array}{c}2015- \\
16\end{array}$ & Pooled & $\begin{array}{c}2014- \\
15\end{array}$ & $\begin{array}{c}2015- \\
16\end{array}$ & Pooled \\
\hline $\mathrm{M}_{1}$ & $\begin{array}{c}0.7 \\
(0.0)\end{array}$ & $\begin{array}{c}0.7 \\
(0.0)\end{array}$ & $\begin{array}{c}0.7 \\
(0.0)\end{array}$ & $\begin{array}{c}0.8 \\
(0.1)\end{array}$ & $\begin{array}{c}0.7 \\
(0.1)\end{array}$ & $\begin{array}{c}0.7 \\
(0.1)\end{array}$ & $\begin{array}{c}0.8 \\
(0.2)\end{array}$ & $\begin{array}{c}0.8 \\
(0.1)\end{array}$ & $\begin{array}{c}0.8 \\
(0.1)\end{array}$ \\
\hline $\mathrm{M}_{2}$ & $\begin{array}{c}0.7 \\
(0.0)\end{array}$ & $\begin{array}{c}0.7 \\
(0.1)\end{array}$ & $\begin{array}{c}0.7 \\
(0.1)\end{array}$ & $\begin{array}{c}0.8 \\
(0.2)\end{array}$ & $\begin{array}{c}0.9 \\
(0.2)\end{array}$ & $\begin{array}{c}0.8 \\
(0.2)\end{array}$ & $\begin{array}{c}1.1 \\
(0.7)\end{array}$ & $\begin{array}{c}0.9 \\
(0.3)\end{array}$ & $\begin{array}{c}1.0 \\
(0.5)\end{array}$ \\
\hline $\mathrm{M}_{3}$ & $\begin{array}{c}0.7 \\
(0.1)\end{array}$ & $\begin{array}{c}0.7 \\
(0.0)\end{array}$ & $\begin{array}{c}0.7 \\
(0.1)\end{array}$ & $\begin{array}{c}0.9 \\
(0.3)\end{array}$ & $\begin{array}{c}0.8 \\
(0.2)\end{array}$ & $\begin{array}{c}0.8 \\
(0.2)\end{array}$ & $\begin{array}{c}1.1 \\
(0.7)\end{array}$ & $\begin{array}{c}0.9 \\
(0.3)\end{array}$ & $\begin{array}{c}1.0 \\
(0.5)\end{array}$ \\
\hline $\operatorname{SEm}( \pm)$ & 0.01 & 0.01 & 0.01 & 0.04 & 0.04 & 0.03 & 0.06 & 0.03 & 0.03 \\
\hline LSD (0.05) & NS & NS & NS & NS & NS & 0.08 & 0.19 & 0.09 & 0.10 \\
\hline & $\begin{array}{c}\text { SEm } \\
( \pm)\end{array}$ & $\begin{array}{c}\text { LSD } \\
(0.05)\end{array}$ & & $\begin{array}{c}\text { SEm } \\
( \pm)\end{array}$ & $\begin{array}{c}\text { LSD } \\
(0.05)\end{array}$ & & $\begin{array}{c}\text { SEm } \\
( \pm)\end{array}$ & $\begin{array}{c}\text { LSD } \\
(0.05)\end{array}$ & \\
\hline Year & 0.01 & NS & & 0.02 & NS & & 0.02 & 0.05 & \\
\hline Year $\times$ Module & 0.01 & NS & & 0.04 & NS & & 0.05 & 0.14 & \\
\hline
\end{tabular}

Note: Data in the parentheses indicate original values. Data shown in the table are $(x+0.5)$ square root transformed values. $\mathrm{M}_{1}$ : IPM module, $\mathrm{M}_{2}$ : Farmers' practices and $\mathrm{M}_{3}$ : Control

Table.6 Effect of management modules on population of thrips per square inch of blue sticky trap installed in onion crop field during rabi, 2014-15 and 2015-16

\begin{tabular}{|c|c|c|c|c|c|c|c|c|c|}
\hline \multirow[t]{3}{*}{ Modules } & \multicolumn{9}{|c|}{ Population of $T$. tabaci per square inch area of blue sticky trap } \\
\hline & \multicolumn{3}{|c|}{ 44DAP } & \multicolumn{3}{|c|}{ 51DAP } & \multicolumn{3}{|c|}{ 58DAP } \\
\hline & $\begin{array}{c}2014- \\
15\end{array}$ & $\begin{array}{c}2015- \\
16\end{array}$ & Pooled & $\begin{array}{c}2014- \\
15\end{array}$ & $\begin{array}{c}2015- \\
16\end{array}$ & Pooled & $\begin{array}{c}2014- \\
15\end{array}$ & $\begin{array}{c}2015- \\
16\end{array}$ & Pooled \\
\hline $\mathbf{M}_{1}$ & $\begin{array}{c}0.9 \\
(0.3)\end{array}$ & $\begin{array}{c}0.8 \\
(0.2)\end{array}$ & $\begin{array}{c}0.9 \\
(0.3)\end{array}$ & $\begin{array}{c}0.9 \\
(0.4)\end{array}$ & $\begin{array}{c}0.8 \\
(0.2)\end{array}$ & $\begin{array}{c}0.9 \\
(0.3)\end{array}$ & $\begin{array}{c}3.6 \\
(12.6)\end{array}$ & $\begin{array}{c}1.5 \\
(1.8)\end{array}$ & $\begin{array}{c}2.5 \\
(7.2)\end{array}$ \\
\hline $\mathrm{M}_{2}$ & $\begin{array}{c}1.3 \\
(1.2)\end{array}$ & $\begin{array}{c}1.1 \\
(0.7)\end{array}$ & $\begin{array}{c}1.2 \\
(0.9)\end{array}$ & $\begin{array}{c}1.8 \\
(2.7)\end{array}$ & $\begin{array}{c}1.2 \\
(0.9)\end{array}$ & $\begin{array}{c}1.5 \\
(1.8)\end{array}$ & $\begin{array}{c}4.2 \\
(17.0)\end{array}$ & $\begin{array}{c}1.2 \\
(1.1)\end{array}$ & $\begin{array}{c}2.7 \\
(9.0)\end{array}$ \\
\hline $\mathrm{M}_{3}$ & $\begin{array}{c}1.3 \\
(1.2)\end{array}$ & $\begin{array}{c}1.0 \\
(0.6)\end{array}$ & $\begin{array}{c}1.2 \\
(0.9)\end{array}$ & $\begin{array}{c}1.8 \\
(2.9)\end{array}$ & $\begin{array}{c}1.6 \\
(2.0)\end{array}$ & $\begin{array}{c}1.7 \\
(2.5)\end{array}$ & $\begin{array}{c}4.3 \\
(18.0)\end{array}$ & $\begin{array}{c}2.0 \\
(3.4)\end{array}$ & $\begin{array}{c}3.1 \\
(10.7)\end{array}$ \\
\hline $\operatorname{SEm}( \pm)$ & 0.05 & 0.04 & 0.03 & 0.03 & 0.06 & 0.03 & 0.08 & 0.09 & 0.06 \\
\hline $\operatorname{LSD}(0.05)$ & 0.16 & 0.13 & 0.11 & 0.09 & 0.19 & 0.11 & 0.25 & 0.26 & 0.18 \\
\hline & $\begin{array}{c}\text { SEm } \\
( \pm)\end{array}$ & $\begin{array}{l}\text { LSD } \\
(0.05)\end{array}$ & & $\begin{array}{l}\text { SEm } \\
( \pm)\end{array}$ & $\begin{array}{c}\text { LSD } \\
(0.05)\end{array}$ & & $\begin{array}{l}\text { SEm } \\
( \pm)\end{array}$ & $\begin{array}{c}\text { LSD } \\
(0.05)\end{array}$ & \\
\hline Year & 0.02 & 0.06 & & 0.02 & 0.07 & & 0.05 & 1.15 & \\
\hline Year $\times$ Module & 0.05 & NS & & 0.05 & 0.14 & & 0.08 & 0.25 & \\
\hline
\end{tabular}

Note: Data in the parentheses indicate original values. Data shown in the table are $(\mathrm{x}+0.5)$ square root transformed values. $\mathrm{M}_{1}$ : IPM module, $\mathrm{M}_{2}$ : Farmers' practices and $\mathrm{M}_{3}$ : Control 
Table.7 Effect of management modules on population of thrips per square inch of blue sticky trap installed in onion crop field during rabi, 2014-15 and 2015-16

\begin{tabular}{|c|c|c|c|c|c|c|c|c|c|}
\hline \multirow[t]{3}{*}{ Modules } & \multicolumn{9}{|c|}{ Population of $T$. tabaci per square inch area of blue sticky trap } \\
\hline & \multicolumn{3}{|c|}{ 65DAP } & \multicolumn{3}{|c|}{ 72DAP } & \multicolumn{3}{|c|}{ 79DAP } \\
\hline & $\begin{array}{c}2014- \\
15\end{array}$ & 2015-16 & Pooled & $\begin{array}{c}2014- \\
15\end{array}$ & 2015-16 & Pooled & $\begin{array}{c}2014- \\
15\end{array}$ & 2015-16 & Pooled \\
\hline $\mathbf{M}_{1}$ & $\begin{array}{c}4.3 \\
(17.8)\end{array}$ & $\begin{array}{c}3.4 \\
(11.1)\end{array}$ & $\begin{array}{c}3.8 \\
(14.5)\end{array}$ & $\begin{array}{c}4.4 \\
(19.3)\end{array}$ & $\begin{array}{c}3.4 \\
(11.4)\end{array}$ & $\begin{array}{c}3.9 \\
(15.3)\end{array}$ & $\begin{array}{c}3.6 \\
(12.8)\end{array}$ & $\begin{array}{c}2.5 \\
(5.9)\end{array}$ & $\begin{array}{c}3.1 \\
(9.4)\end{array}$ \\
\hline $\mathbf{M}_{2}$ & $\begin{array}{c}3.6 \\
(12.9)\end{array}$ & $\begin{array}{c}3.0 \\
(8.3)\end{array}$ & $\begin{array}{c}3.3 \\
(10.6)\end{array}$ & $\begin{array}{c}4.4 \\
(18.8)\end{array}$ & $\begin{array}{c}3.3 \\
(10.7)\end{array}$ & $\begin{array}{c}3.9 \\
(14.8)\end{array}$ & $\begin{array}{c}3.7 \\
(13.0)\end{array}$ & $\begin{array}{c}2.9 \\
(8.2)\end{array}$ & $\begin{array}{c}3.3 \\
(10.6)\end{array}$ \\
\hline $\mathbf{M}_{3}$ & $\begin{array}{c}4.4 \\
(18.7)\end{array}$ & $\begin{array}{c}3.5 \\
(12.0)\end{array}$ & $\begin{array}{c}3.9 \\
(15.3)\end{array}$ & $\begin{array}{c}4.0 \\
(15.4)\end{array}$ & $\begin{array}{c}4.0 \\
(15.4)\end{array}$ & $\begin{array}{c}4.0 \\
(15.4)\end{array}$ & $\begin{array}{c}4.2 \\
(17.1)\end{array}$ & $\begin{array}{c}4.0 \\
(15.8)\end{array}$ & $\begin{array}{c}4.1 \\
(16.4)\end{array}$ \\
\hline $\operatorname{SEm}( \pm)$ & 0.13 & 0.07 & 0.07 & 0.04 & 0.10 & 0.05 & 0.04 & 0.15 & 0.08 \\
\hline LSD (0.05) & 0.40 & 0.21 & 0.22 & 0.12 & 0.29 & NS & 0.12 & 0.48 & 0.24 \\
\hline & $\begin{array}{c}\text { SEm } \\
( \pm)\end{array}$ & $\begin{array}{c}\text { LSD } \\
(0.05)\end{array}$ & & $\begin{array}{c}\text { SEm } \\
( \pm)\end{array}$ & $\begin{array}{c}\text { LSD } \\
(\mathbf{0 . 0 5})\end{array}$ & & $\begin{array}{c}\text { SEm } \\
( \pm)\end{array}$ & $\begin{array}{l}\text { LSD } \\
(\mathbf{0 . 0 5})\end{array}$ & \\
\hline Year & 0.05 & 0.15 & & 0.03 & 0.11 & & 0.07 & 0.20 & \\
\hline Year×Module & 0.10 & NS & & 0.07 & 0.22 & & 0.11 & 0.33 & \\
\hline
\end{tabular}

Note: Data in the parentheses indicate original values. Data shown in the table are $(x+0.5)$ square root transformed values. $\mathrm{M}_{1}$ : IPM module, $\mathrm{M}_{2}$ : Farmers' practices and $\mathrm{M}_{3}$ : Control

Table.8 Effect of management modules on population of thrips per square inch of blue sticky trap installed in onion crop field during rabi, 2014-15 and 2015-16

\begin{tabular}{|c|c|c|c|c|c|c|c|c|c|}
\hline \multirow[t]{3}{*}{ Modules } & \multicolumn{9}{|c|}{ Population of $T$. tabaci per square inch area of blue sticky trap } \\
\hline & \multicolumn{3}{|c|}{ 86DAP } & \multicolumn{3}{|c|}{ 93DAP } & \multicolumn{3}{|c|}{ 100DAP } \\
\hline & $\begin{array}{c}2014- \\
15\end{array}$ & $\begin{array}{c}2015- \\
16\end{array}$ & Pooled & $\begin{array}{c}\text { 2014- } \\
15\end{array}$ & $\begin{array}{l}2015- \\
16\end{array}$ & Pooled & $\begin{array}{c}2014- \\
15\end{array}$ & $\begin{array}{c}2015- \\
16\end{array}$ & Pooled \\
\hline $\mathbf{M}_{1}$ & $\begin{array}{c}3.3 \\
(10.7)\end{array}$ & $\begin{array}{c}4.3 \\
(17.6)\end{array}$ & $\begin{array}{c}3.8 \\
(14.2)\end{array}$ & $\begin{array}{c}2.5 \\
(5.6)\end{array}$ & $\begin{array}{c}2.8 \\
(7.6)\end{array}$ & $\begin{array}{c}2.6 \\
(6.6)\end{array}$ & $\begin{array}{c}2.1 \\
(4.1)\end{array}$ & $\begin{array}{c}2.2 \\
(4.2)\end{array}$ & $\begin{array}{c}2.1 \\
(4.1)\end{array}$ \\
\hline $\mathbf{M}_{2}$ & $\begin{array}{c}4.4 \\
(18.6)\end{array}$ & $\begin{array}{c}4.3 \\
(18.1)\end{array}$ & $\begin{array}{c}4.3 \\
(18.4)\end{array}$ & $\begin{array}{c}2.9 \\
(8.1)\end{array}$ & $\begin{array}{c}3.2 \\
(9.5)\end{array}$ & $\begin{array}{c}3.0 \\
(8.8)\end{array}$ & $\begin{array}{c}2.7 \\
(7.1)\end{array}$ & $\begin{array}{c}2.6 \\
(6.4)\end{array}$ & $\begin{array}{c}2.7 \\
(6.7)\end{array}$ \\
\hline $\mathbf{M}_{3}$ & $\begin{array}{c}4.3 \\
(17.8)\end{array}$ & $\begin{array}{c}5.1 \\
(26.1)\end{array}$ & $\begin{array}{c}4.7 \\
(22.0)\end{array}$ & $\begin{array}{c}3.8 \\
(13.9)\end{array}$ & $\begin{array}{c}5.2 \\
(27.0)\end{array}$ & $\begin{array}{c}4.5 \\
(20.5)\end{array}$ & $\begin{array}{c}3.1 \\
(9.4)\end{array}$ & $\begin{array}{c}3.7 \\
(13.3)\end{array}$ & $\begin{array}{c}3.4 \\
(11.4)\end{array}$ \\
\hline $\operatorname{SEm}( \pm)$ & 0.04 & 0.15 & 0.08 & 0.14 & 0.13 & 0.09 & 0.10 & 0.05 & 0.06 \\
\hline LSD (0.05) & 0.12 & 0.46 & 0.24 & 0.43 & 0.39 & 0.29 & 0.31 & 0.14 & 0.17 \\
\hline & $\begin{array}{c}\text { SEm } \\
( \pm)\end{array}$ & $\begin{array}{c}\text { LSD } \\
(\mathbf{0 . 0 5})\end{array}$ & & $\begin{array}{c}\text { SEm } \\
( \pm)\end{array}$ & $\begin{array}{c}\text { LSD } \\
(0.05)\end{array}$ & & $\begin{array}{c}\text { SEm } \\
( \pm)\end{array}$ & $\begin{array}{c}\text { LSD } \\
(\mathbf{0 . 0 5})\end{array}$ & \\
\hline Year & 0.07 & 0.21 & & 0.07 & 0.21 & & 0.06 & NS & \\
\hline Year×Module & 0.11 & 0.32 & & 0.13 & 0.39 & & 0.08 & 0.23 & \\
\hline
\end{tabular}

Note: Data in the parentheses indicate original values. Data shown in the table are $(x+0.5)$ square root transformed values. $\mathrm{M}_{1}$ : IPM module, $\mathrm{M}_{2}$ : Farmers' practices and $\mathrm{M}_{3}$ : Control 
Table.9 Effect of thrips management modules on number of onion bulbs during rabi, 2014-15 and 2015-16

\begin{tabular}{|c|c|c|c|}
\hline \multirow{2}{*}{ Modules } & \multicolumn{3}{|c|}{ Number of bulbs/plot } \\
\cline { 2 - 4 } & $\mathbf{2 0 1 4 - 1 5}$ & $\mathbf{2 0 1 5 - 1 6}$ & Pooled \\
\hline $\mathbf{M}_{\mathbf{1}}$ & 122 & 184 & 153 \\
\hline $\mathbf{M}_{\mathbf{2}}$ & 119 & 179 & 149 \\
\hline $\mathbf{M}_{\mathbf{3}}$ & 108 & 174 & 141 \\
\hline SEm $( \pm)$ & $\mathbf{2 . 7 2}$ & $\mathbf{2 . 5 4}$ & $\mathbf{1 . 8 6}$ \\
\hline LSD $(\mathbf{0 . 0 5})$ & $\mathbf{8 . 3 8}$ & $\mathbf{7 . 8 3}$ & $\mathbf{5 . 7 3}$ \\
\hline & $\mathbf{S E m}( \pm)$ & $\mathbf{L S D}(\mathbf{0 . 0 5})$ & \\
\hline Year & $\mathbf{0 . 3 0}$ & $\mathbf{0 . 9 2}$ & \\
\hline Year×Module & $\mathbf{2 . 6 3}$ & $\mathbf{N S}$ & \\
\hline
\end{tabular}

Table.10 Effect of thrips management modules on fresh weight of onion bulb during rabi, 201415 and 2015-16

\begin{tabular}{|c|c|c|c|c|c|c|}
\hline \multirow[t]{2}{*}{ Modules } & \multicolumn{3}{|c|}{$\begin{array}{c}\text { Fresh weight of bulb } \\
\text { (kg/plot) }\end{array}$} & \multicolumn{3}{|c|}{$\begin{array}{c}\text { Fresh weight of bulb } \\
\text { (q/ha) }\end{array}$} \\
\hline & 2014-15 & 2015-16 & Pooled & 2014-15 & $2015-16$ & Pooled \\
\hline $\mathbf{M}_{1}$ & 8.57 & 13.04 & 10.81 & $226.73^{*}$ & $316.87^{*}$ & 271.80 \\
\hline $\mathbf{M}_{2}$ & 7.97 & 12.56 & 10.27 & 194.70 & 306.72 & 250.70 \\
\hline $\mathbf{M}_{3}$ & 7.05 & 11.49 & 9.27 & 172.11 & 280.67 & 226.39 \\
\hline $\operatorname{SEm}( \pm)$ & 0.18 & 0.18 & 0.13 & 3.90 & 3.64 & 2.67 \\
\hline \multirow[t]{2}{*}{ LSD (0.05) } & 0.55 & 0.55 & 0.40 & 12.02 & 11.22 & 8.22 \\
\hline & SEm ( \pm$)$ & LSD (0.05) & & SEm ( \pm ) & LSD (0.05) & \\
\hline Year & 0.02 & 0.06 & & 0.63 & 1.94 & \\
\hline Year×Module & 0.18 & NS & & 3.78 & 11.62 & \\
\hline
\end{tabular}

Note: Onion equivalent yield was calculated considering market price of onion as Rs. 400/q, wheat grain as Rs. $1450 / \mathrm{q}$ and maize grain as Rs. 1500/q

Table.11 Effect of thrips management modules on dry weight of onion bulb during rabi, 201415 and 2015-16

\begin{tabular}{|c|c|c|c|c|c|c|}
\hline \multirow[t]{2}{*}{ Modules } & \multicolumn{3}{|c|}{$\begin{array}{c}\text { Dry weight of bulb } \\
\text { (kg/plot) }\end{array}$} & \multicolumn{3}{|c|}{$\begin{array}{c}\text { Dry weight of bulb } \\
\text { (q/ha) }\end{array}$} \\
\hline & 2014-15 & 2015-16 & Pooled & 2014-15 & $2015-16$ & Pooled \\
\hline $\mathbf{M}_{1}$ & 8.07 & 12.45 & 10.26 & $218.15^{*}$ & $306.74^{*}$ & 262.54 \\
\hline $\mathbf{M}_{2}$ & 7.51 & 11.98 & 9.75 & 183.47 & 292.55 & 238.01 \\
\hline $\mathbf{M}_{3}$ & 6.50 & 10.92 & 8.71 & 158.69 & 266.75 & 212.72 \\
\hline $\operatorname{SEm}( \pm)$ & 0.19 & 0.18 & 0.13 & 4.07 & 3.64 & 2.73 \\
\hline LSD (0.05) & 0.58 & 0.55 & 0.40 & 12.55 & 11.22 & 8.42 \\
\hline & SEm ( \pm$)$ & $\operatorname{LSD}(0.05)$ & & $\operatorname{SEm}( \pm)$ & $\operatorname{LSD}(0.05)$ & \\
\hline Year & 0.03 & 0.09 & & 0.80 & 2.47 & \\
\hline Year×Module & 0.18 & NS & & 3.86 & 11.90 & \\
\hline
\end{tabular}

Note: Onion equivalent yield was calculated considering market price of onion as Rs. $500 / \mathrm{q}$, wheat grain as Rs. $1450 / \mathrm{q}$ and maize grain as Rs. $1500 / \mathrm{q}$, mean grain yield of wheat and maize were $5.87 \mathrm{q}$ and $22.78 \mathrm{q}$ in $3030 \mathrm{sq} . \mathrm{m}$ area 
Table.12 Estimation of avoidable yield loss of onion bulb due to T. tabaci

\begin{tabular}{|l|c|}
\hline \multicolumn{1}{|c|}{ Module } & \% avoidable yield loss \\
\hline $\mathbf{M}_{\mathbf{1}}$ : IPM Module & 18.98 \\
\hline $\mathbf{M}_{3}$ : Untreated Control & -- \\
\hline
\end{tabular}

Table.13 Comparative economics of adopted thrips management modules for rabi onion based on pooled dry bulb yield (i.e. marketable yield) data

\begin{tabular}{|c|c|c|c|c|c|c|c|c|c|}
\hline \multirow[t]{2}{*}{ Modules } & \multirow{2}{*}{$\begin{array}{c}* \text { Onion } \\
\text { dry } \\
\text { bulb } \\
\text { yield } \\
(\mathbf{q} / \mathrm{ha})\end{array}$} & \multirow{2}{*}{$\begin{array}{c}\text { Gain in } \\
\text { bulb } \\
\text { yield } \\
\text { over } \\
\text { control } \\
\text { (q/ha) }\end{array}$} & \multirow[t]{2}{*}{$\begin{array}{l}\text { Value of additional yield } \\
\text { (Rs./ha) }\end{array}$} & \multicolumn{4}{|c|}{$\begin{array}{l}\text { Cost of treatment } \\
\text { (Rs./ha) }\end{array}$} & \multirow[t]{2}{*}{$\begin{array}{l}\text { Net gain } \\
\text { (Rs./ha) }\end{array}$} & \multirow[t]{2}{*}{ IBCR } \\
\hline & & & & Chemical & Labour & Others ${ }^{\#}$ & Total & & \\
\hline $\mathbf{M}_{1}$ & $262.54^{* *}$ & 49.82 & 24910.00 & 931.00 & 915.00 & 1900.00 & 3746.00 & 21164.00 & 6.65 \\
\hline $\mathbf{M}_{2}$ & 238.01 & 25.29 & 12645.00 & 671.00 & 1332.00 & -- & 2003.00 & 10642.00 & 6.31 \\
\hline $\mathbf{M}_{3}$ & 212.72 & -- & -- & -- & -- & -- & -- & -- & -- \\
\hline
\end{tabular}

Note: * pooled data, ** onion equivalent yield, labour wages-Rs. 222/ man-day, market price of onion bulb- Rs. 500/q, market price of Beauvariabassiana10SC-Rs.600/litre, acetamiprid20SP-Rs. 1200/kg, imidacloprid 17.8SL-Rs.1300/litre, imidacloprid 70WS-Rs. 3000/kg, acephate 75 SP-Rs.550/kg, spinosad 45SCRs.4800/litre, \#include price of wheat and maize seeds including labour wagesfor sowing of seeds in row, $\mathrm{IBCR}=$ incremental benefit cost ratio

Significant variations on number of bulbs/plot between years were observed here. The interaction effect between thrips management module and year of study on number of bulbs/plot was found non-significant. The data presented in the table- 10 indicated the effect of modules on fresh weight of onion bulb during 2014-15 and 2015-16. Significantly maximum fresh weight of onion bulb was observed with $\mathrm{M}_{1}$ being, 226.73 $\mathrm{q} / \mathrm{ha}$ and followed by $\mathrm{M}_{2}$ being, $194.70 \mathrm{q} / \mathrm{ha}$ during 2014-15.

During the second year of study (2015-16), maximum fresh weight of onion bulb was also observed in $\mathrm{M}_{1}$ being, $316.87 \mathrm{q} / \mathrm{ha}$ and was followed by $\mathrm{M}_{2}$ being, $306.72 \mathrm{q} / \mathrm{ha}$. Both the modules were at par with each other but significantly superior to untreated control. Based on pooled data, the maximum yield was $271.80 \mathrm{q} / \mathrm{ha}$ in IPM module whereas, farmers' practice module had $250.70 \mathrm{q} / \mathrm{ha}$ of fresh bulb yield (Table 10). Significant variations in fresh bulb yield of onion between years were observed. The interaction effect between thrips management module and year of study on fresh yield of onion bulbs was found significant.

Unlike fresh bulb yield, the dry bulb yield of onion was recorded maximum with IPM module in both the years being, 218.15 and $306.74 \mathrm{q} / \mathrm{ha}$ for the $1^{\text {st }}$ and $2^{\text {nd }}$ year, respectively (Table 11). Performance of farmers' practice module was just after IPM module in achieving dry yield of onion bulb. Considering, pooled data maximum dry yield of onion bulb was documented in IPM module being, $262.54 \mathrm{q} / \mathrm{ha}$ (Table 11). Dry onion bulb yield in farmers' practice module was $238.01 \mathrm{q} / \mathrm{ha}$.

Similar observation was also reported in previous study where IPM module achieved more bulb production of onion over the farmers' practice and untreated control 
Int.J.Curr.Microbiol.App.Sci (2018) 7(7): 2865-2877

modules (Tripathy et al., 2013). 
Estimation of avoidable yield loss of onion bulb due to $T$. tabaci

An estimation of avoidable yield loss of onion bulb due to infestation of $T$. tabaci was made considering pooled yield data (i.e. onion equivalent yield) of IPM module and untreated control module (Table 12). The analysis revealed that adoption of IPM module suitably checked $18.98 \%$ loss of onion blub yield due to T. tabaci infestation (Table 12).

The maximum total bulb and marketable bulb yield losses of 16.9 and $17.7 \mathrm{t} / \mathrm{ha}$, respectively were observed with December transplanted onion crop by Merene (2015). The yield loss estimation by the present author remains in parity with Merene (2015).

Less insecticidal application was the reason for having higher benefit cost ratio in IPM module. The additional income was also obtained from the border crop like maize and wheat in the IPM module. Tripathy et al., (2013) also got the highest benefit cost ratio in IPM module of thrips management than that of farmers' practice. Observation of the present study remains in parity with the findings of Tripathy et al., (2013).

\section{References}

Anjaneyulu, V.R., S.P. Singh and M. Pal. 1982. Effect of competition free period and technique and pattern of pearlmillet planting on growth and yield of mungbean and total productivity in solid pearlmillet / mungbean intercropping system. Indian Journal of Agronomy, 27: 219226.

Annamalai, M., Kaushik, H. D. and Selvaraj, K. 2016. Bioefficacy of Beauveria bassiana (Balsamo) Vuillemin and Lecanicillium lecanii Zimmerman against Thrips tabaci Lindeman. Proceedings of the National Academy of Sciences, India Section B, Biological Sciences, 86(2): 505-511.

Basavaraju, B. S., Chakravarthy, A. K., Doddabasappa, B. and Krishnamurthy, N. 2009. Yield loss estimation due to major insect and mite pests on potato in Karnataka. Karnataka Journal of Agricultural Sciences, 22 (3-Spl. Issue): 597-600.

Brian, A. N. 2006. Biology and ecology of onion thrips on onion fields. Cornell University Report, pp. 2.

Darmasena, C. M. D. 1998. Present status of managing leaf curl complex in the north central province of Sri Lanka. Tropical Agricultural Research and Extension, 1: 154-158.

Diaz-Montano, J., Fuchs, M., Nault, B. A., Fail, J. and Shelton, A. M. 2011. Onion thrips (Thysanoptera: Thripidae): A global pest of increasing concern in onion. Journal of Economic Entomology, 104: 1-13.

Gomez, K.A. and Gomez, A.A. 1984. Statistical Procedures for Agricultural Research (2 ${ }^{\text {nd }}$ Ed.). John Wiley and Sons, New York, pp. 704.

Gupta, R. P., Srivastava, V. K., Bhardwaj, B. S. and Pandey, U. B. 1984. Chemical control of Thrips tabaci Lind. infesting onion crop. Journal of Entomological Research, 8(2): 196198.

http://skuast.org/ site/ Templates\%20 HTML/research /seed-img/ seed.pdf accessed on 20.09. 2014.

http://www.dogr.res.in/ index.php? option=com_content \& view = article\&id $=97 \&$ accessed on 14.12.2013

Mahmoud, M. F. and Osman, M. A. M. 2007. Relative toxicity of some bio-rational insecticides to second instar larvae and adults of onion thrips (Thrips tabaci 
Lind.) and their predator Orius albidipennis under laboratory and field conditions. Journal of Plant Protection Research, 47 (4):391-400.

Merene, Y. 2015. Population dynamics and damages of onion thrips (Thrips tabaci) (Thysanoptera: Thripidae) on onion in Northeastern Ethiopia. Journal of Entomology and Nematology, 7(1): 1-4.

Panse, R. K., Gupta, A. and Jain, P. K. 2012. Eco-friendly management of Thrips tabaci (Lindeman) in onion. Pesticide Research Journal, 24(2):155-158.

Roy, K., Mukhopadhyay, A. K. and Pramanik, A. 2008. Assessment of Yield Loss due to Rotylenchulus reniformis Linford \& Oliveira, 1940 in Cowpea, Vigna unguiculata (L.) Walp. Annals of Plant Protection Sciences, 16 (1): 191-193.

Sudharma, K. and Nair, G. M. 1999. Assessment of loss caused by Polyphagotarsonemus latus (Banks) on chilli. Entomon, 24(1): 97-98.

Talukder, A.H.M.M.R., Rahman, J., Nahar, L., Rahman, M. M. and Kaisar, N. 2015. Mixed Cropping Onion with Different Plant Population of Sweet Gourd. IOSR Journal of Agriculture and Veterinary Science, 8 (5): 45-50.

Tripathy, P., Sahoo, B. B., Patel, D., Das, S. K., Priyadarshini, A. and Dash, D. K. 2013. Validation of IPM module against onion thrips Thrips tabaci Lindeman. Indian Journal of Entomology, 75(4): 298-300.

\section{How to cite this article:}

Maimom Soniya Devi and Kusal Roy. 2018. An Integrated Approach for Management of Thrips tabaci Lindeman in Rabi Onion under Gangetic Plains of West Bengal, India. Int.J.Curr.Microbiol.App.Sci. 7(07): 2865-2877. doi: https://doi.org/10.20546/ijcmas.2018.707.336 